\title{
Support system for ATLAS distributed computing operations
}

\author{
Tomoe Kishimoto*, on behalf of the ATLAS Collaboration \\ International Center for Elementary Particle Physics, The University of Tokyo \\ E-mail: Tomoe.Kishimotodcern.ch
}

The ATLAS distributed computing system has allowed the experiment to successfully meet the challenges of LHC Run 2. In order for distributed computing to operate smoothly and efficiently, several support teams are organized in the ATLAS experiment. The ADCoS is a dedicated group of shifters who follow and report failing jobs, failing data transfers between sites, degradation of ATLAS central computing services, and more. The DAST provides user support to resolve issues related to running distributed analysis on the Grid. The CRC maintains a global view of the dayto-day operations. In this paper, the status and operational experience of the support system for ATLAS distributed computing in LHC Run 2 are reported. This report also includes operations experience from the Grid site point of view, and an analysis of the errors that create the biggest waste of wallclock time. The report of operations experience will focus on some of the more time-consuming tasks for shifters, and on the introduction of new technologies, such as machine learning, to ease the work.

The 39th International Conference on High Energy Physics (ICHEP2018)

4-11 July, 2018

Seoul, Korea

${ }^{*}$ Speaker. 


\section{Introduction}

The ATLAS experiment [ $[$ ] at the LHC produces a large amount of data to cover various physics analyses. In order to process and store such a large data, computing resources are distributed around the world. The distributed computing resources are managed by the ATLAS Distributed Computing (ADC) system, which is composed of several services such as the workload management system, the distributed data management system and so on.

\section{Support teams in ATLAS and monitoring tools}

Several types of shift teams are organized to support the ADC operation. The ATLAS Distributed Computing Shift (ADCoS) follows and reports failing jobs, failing transfers, degradation of ATLAS central computing services, and more. The Distributed Analysis Support Team (DAST) provides user support to resolve issues related to running distributed analysis on the Grid. The Computing Run Coordinator (CRC) maintains a global view of day-to-day operation. The shifters use various tools to monitor the status of the ADC operations. For example, the tools for ADCoS shifters are summarized on a web page [[]].

\section{Operation experience in LHC Run2 (2015-2018)}

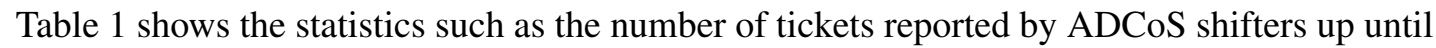
July 2018 in LHC-Run2. For example, problems of Grid sites are well reported by the shifters. File transfer and deletion errors were the main reason for Global Grid User Support (GGUS) tickets to Grid sites. $92 \%$ of the GGUS tickets were solved within three days. The job error that creates the biggest waste of wallclock time was "lost heartbeat" error in 2018 . The most common reason for the error is that the job was killed by the local batch system because it used more than the allowed resources.

\begin{tabular}{ccc|c}
\hline $\begin{array}{c}\text { \# of tickets to sites } \\
\text { (GGUS system) }\end{array}$ & $\begin{array}{c}\text { \# of tickets to ATLAS } \\
\text { (JIRA system) }\end{array}$ & \# of e-log entries & DAST of e-mails from users \\
\hline \hline$\sim 2500$ & $\sim 850$ & $\sim 11000$ & $\sim 35000$ \\
\hline
\end{tabular}

Table 1: The number of tickets, e-log entries and e-mails handled by the shifters.

\section{Discussions}

Automation is a key for the future ADC operation because the personpower is limited and understanding errors and ticketing are time-consuming tasks. Machine learning is a promising approach to ease the shifter's work. A possible future plan is that we build a framework, which could learn shifter's decisions based on metrics, and then provide notification or automated resolution.

\section{References}

[1] ATLAS Collaboration, 2008 JINST 3 S08003.

[2] Monitoring tools for ADCoS shifter: https://adcos.web.cern.ch/adcos/For_ADCoS_Shifters/ 